\title{
Low-cost fiber specklegram sensor for noncontact continuous patient monitoring
}

Alberto Rodríguez-Cuevas

Eusebio Real Peña

Luis Rodríguez-Cobo

Mauro Lomer

José Miguel López Higuera 


\title{
Low-cost fiber specklegram sensor for noncontact continuous patient monitoring
}

\author{
Alberto Rodríguez-Cuevas, ${ }^{a, *}$ Eusebio Real Peña, ${ }^{a}$ Luis Rodríguez-Cobo, ${ }^{\mathrm{a}, \mathrm{b}}$ Mauro Lomer, ${ }^{\mathrm{a}, \mathrm{b}}$ and \\ José Miguel López Higuera ${ }^{\mathrm{a}, \mathrm{b}}$ \\ aUniversity of Cantabria, Photonics Engineering Group, Santander, Spain \\ ${ }^{\mathrm{b}} \mathrm{CIBER}-\mathrm{BBN}$, Instituto de Salud Carlos III, Madrid, Spain
}

\begin{abstract}
Different low-cost approaches based on fiber specklegram sensors are employed to measure the physiological activity of patients lying in bed. This study is conducted over 20 volunteers to measure the movement and heart rate (HR) using two different sensor designs placed at two different bed locations. Three different processing methods are developed and tested in order to extract useful information from the measured data. The results suggest that a stretched fiber optic configuration under the head of the person lying in bed is the optimal configuration to detect HR and motion, nonetheless the other tested possibilities also exhibit remarkably good performances. On the other hand, the three proposed processing methods also achieve a good precision in the HR detection. The sensor implementation is simple, not requiring any special conditions, and it provides robust performance. This leads to the conclusion that fiber specklegram technology is a feasible method to be used in real situations for heartbeat and movement monitoring. @ 2017 Society of Photo-Optical Instrumentation Engineers (SPIE) [DOI: 10 .1117/1.JBO.22.3.037001]
\end{abstract}

Keywords: speckle; monitoring; vital sings; optics; fiber optic; noninvasive.

Paper 160634RR received Sep. 14, 2016; accepted for publication Feb. 10, 2017; published online Mar. 2, 2017.

\section{Introduction}

\subsection{Background}

Technology for noncontact monitoring techniques has been studied for more than 50 years. ${ }^{1}$ During the last decade, the medical community and authorities from different care systems around the world have started thinking of noncontact monitoring as a necessary tool for present and future medicine.

At present, 24-h monitoring scenarios only take place in the intensive care unit (ICU), even in the most developed countries. Nonetheless, several studies have found strong evidence that continuous monitoring methods, compared with conventional ones, can be more effective against health deterioration of hospitalized patients, especially in those cases related to heart conditions. ${ }^{2,3}$

In most cases, cardiac arrest is preceded by abnormal breathing and heartbeat frequencies, indicating that it could be detected on time before it happens. In such a situation, patients could be transferred from their regular hospital rooms to the better equipped ICU in order to proceed to a further health evaluation. Eventually, the average time that patients need to recover, as well as long-term economic costs, may be reduced.

In terms of patient monitoring, current methods have some disadvantages, which make them inconvenient for continuous vital signs monitoring. The need of sticking sensors to the skin reduces the mobility of the patient and may be uncomfortable to the patient. Moreover, sensors need to be detached and reattached each time the inpatient needs to be moved, even if it is for a short period of time. Considering these facts, noncontact monitoring technologies are being developed in different universities and research centers worldwide. The most

*Address all correspondence to: Alberto Rodríguez-Cuevas, E-mail: alberto rodriguez@unican.es studied methods include ballistocardiography, capacitive electrocardiogram, infrared thermography, magnetic induction monitoring, or photoplethysmography imaging. ${ }^{4-7}$

Lately, the fiber-optics-based techniques have grown in importance within the noncontact monitoring field due to their versatility and their possibility of being used with singular environments, such as magnetic resonance imaging scans. In these environments, placing the metal or common electronic components is not an option as it can cause them to heat up and malfunction. ${ }^{8}$

Among fiber optics techniques, the speckle technology has emerged as the most promising method for monitoring heartbeat and motion due to its high sensitivity and relatively low cost. In this regard, one of the first tests was carried out in the University of Virginia in 2004. It concludes that these types of sensors have the potential to become a cost-effective method of automating long-term monitoring of patients. ${ }^{9}$ Since that test, similar experiments have been carried out in different institutions. ${ }^{10,11}$

In this work, different speckle-based optical-fiber sensors are employed to detect and measure heart rate (HR) and motion of patients lying on a bed. The proposed devices are able to measure HR and motion without direct contact with people's skin, even when lying in different positions. The achieved results verify the applicability of these kinds of sensors to be employed in real scenarios.

\subsection{Speckle in Multimode Fiber}

Speckle phenomenon in fiber optics is generated by the spread of a large number of modes with different phase velocities that is given when a coherent light is propagated through a multimode fiber. The propagation modes corresponding to different optical

1083-3668/2017/\$25.00 @ 2017 SPIE 


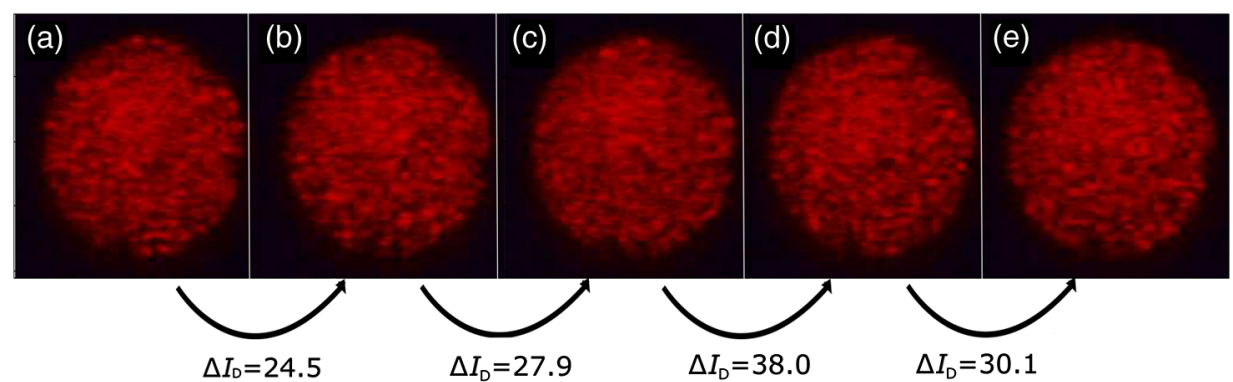

Fig. 1 Example of a five-frame sequence caption and the frame-to-frame differences obtained by the described method [Eq. (5)] under intense perturbations: (a) frame 50, (b) frame 51, (c) frame 52, (d) frame 53 , and (e) frame 54 .

paths (used by the beams coupled into the fiber) suffer different phase delays. The output field distribution consists of the sum of all individual contributions of each mode. If the phase delay contribution varies over $2 \pi$ radians, having a sufficiently coherent source, then interference effects are well structured and can be observed in the intensity distribution through the end of the fiber. ${ }^{12}$ The number of modes, $M$, that supports an optical fiber break index is given by Eq. (1). These modes interfere among themselves and also individually in a self-mode interaction. ${ }^{13}$

$M=\frac{V^{2}}{2}$,

where $V$ is called a normalized frequency given by

$V=\frac{2 a \pi}{\lambda} \sqrt{n_{c o}^{2}-n_{c l}^{2}}$,

where $a$ is the core radius, $\lambda$ is the wavelength of the laser, and $n_{c o}$ and $n_{c l}$ are the refractive indices of core and shell, respectively.

The output speckle pattern projected from the end of the fiber (specklegram) is composed of a large number of individual speckles (bright dots and dark areas). The intensity of each individual speckle may vary, while the total intensity of the speckle pattern must remain constant.

Particularly, perturbations applied to optical fibers have an influence on phase, polarization, and distribution of the modes. By analyzing variations within speckle patterns, different perturbations applied to the medium (e.g., optical fiber) can be detected, among them vibration, ${ }^{14}$ displacement, ${ }^{15}$ angular alignment, ${ }^{16}$ strain, ${ }^{17,18}$ and even vital signs. ${ }^{9-11}$

An approximated relation between the perturbations and the variations of speckle patterns can be directly obtained to get information regarding the applied perturbation, simplifying the final model. Indeed, there is a model ${ }^{14}$ that determines the relation between the speckle pattern variation and the perturbation to be measured. This model considers that the mode propagation and the mode interference are both modified by the perturbation, $F(t)$, which is reflected in the speckle intensity of each speckle, $I_{i}$. This parameter can be calculated as the integration of the spatial intensity function for each speckle area as is shown below

$I_{i}=A_{i} *\left\{1+B_{i} *\left[\cos \left(\delta_{i}\right)-F(t) * \phi_{i} * \sin \left(\delta_{i}\right)\right]\right\}$,

where $A_{i}$ represents the self-mode interaction, and $F(t), B_{i}$, and $\delta_{i}$ define the interaction between different modes. Particularly,
$F(t)$ is the external perturbation of the fiber. The argument of the harmonic functions $\delta_{i}$ describes the difference in the propagation constant and the random phase of all the pairs of modes considered within the same speckle intensity. $A_{i}, B_{i}, \phi_{i}$, and $\delta_{i}$ are constant values for any given $i$.

In order to extract this information, $F(t)$, a differential processing method is applied to Eq. (3). The sum of the absolute value of the changes in all the signals is computed as $\left(\Delta I_{T}\right)$ based on the pixel sensitivity $(C)$ and can be described by

$\Delta I_{T}=C * \frac{d F(t)}{d t}=C * \Delta F$.

Based on this approach, a differential processing method can be applied to determine the relation between the speckle pattern variation and the external perturbation measured $\left(\Delta I_{D}\right)$ for every $i$ 'th pixel

$\Delta I_{D}\{i\}=\frac{1}{K * M N} \sum_{n=0}^{N-1} \sum_{m=0}^{M-1}\left|I_{n m}^{i-1}-I_{n m}^{i}\right|$,

where $K$ is the full scale value of the speckle pattern color map (e.g., $K=255$ for a 8-bit grayscale), and $I_{n m}^{i}$ corresponds to the pixel of the $n, m$ (considering $N \times M$ pixels) position of the $i$ 'th speckle pattern. The relation described by Eq. (5) assumes that the total intensity of the speckle patterns remains constant under perturbations, being the computed value, an amount that quantifies the power migration between individual speckles within the pattern.

With this differential processing method, dynamic measurements can be obtained and are mainly limited by the sampling rate of the specific CCD camera of the specklegram sensor. The sensitivity of this fiber specklegram sensor ["C" in Eq. (4)] is basically given by the amount and contrast of the dots (individual speckles) comprised within the captured images and can be determined by a proper sizing of the laser source, multimode fiber, and detector.

In Fig. 1, five consecutive specklegrams are displayed, corresponding to a sequence in which a $240-\mu$ m-core fiber optic was under intense external perturbations caused by human motion.

\section{Materials and Methods}

In order to detect vital signs of patients lying in bed, a prospective study was carried out so as to set the basic conditions for the experiments. Initially, three different diameters of polymer optical fibers (POF) were tested: 50, 240, and $980 \mu \mathrm{m}$, in 

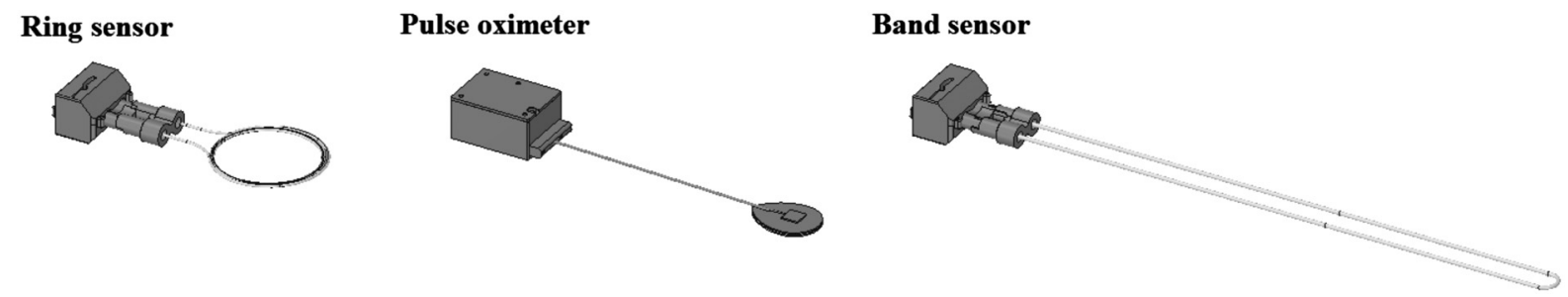

Fig. 2 3-D representation of the sensors used in the experiment.

combination with several different support materials, such as thin wooden boards, silicone supports, soft plastics, bare fiber, cotton fabric, and other textiles. Later, several fiber arrangement geometries were tested as well, such as ring, helix, or straight fiber. Based on sensitivity and noise compromise, two sensors were selected. Hereafter, we will refer to these sensors as band sensor and ring sensor. Both sensors consisted of a semiconductor laser emitting at $638-\mathrm{nm}$ wavelength, a CCD camera that produces data at a rate of 30 frames per second, and a plastic fiber optic that was $200 \mathrm{~cm}$ long and had a $240-\mu$ m-diameter core. The core diameter of the fiber and the laser wavelength were selected to optimize sensitivity for the application.

In the case of the band sensor, the POF was set inside two lateral seams of cotton fabric $7-\mathrm{cm}$ wide and $80-\mathrm{cm}$ long. In the case of the ring sensor, the POF was rolled creating 10 laps of an average diameter of $6 \mathrm{~cm}$ and was set inside two cotton fabrics of $10 \mathrm{~cm}$ on each side (Fig. 2). A conventional pulse oximeter was used to obtain reference values of the heartbeats in all the measurements. It was held with the thumb and the index finger by the person under analysis during each test.

Twenty healthy volunteers selected and provided their informant consent, and the tests were approved by the University of Cantabria Ethics Committee and the Photonics Engineering Group. Both the ring sensor and the band sensor recorded measurements simultaneously with the pulse oximeter. Four different 160-s-long measurements were taken from each volunteer with both the sensors: two measurements were taken
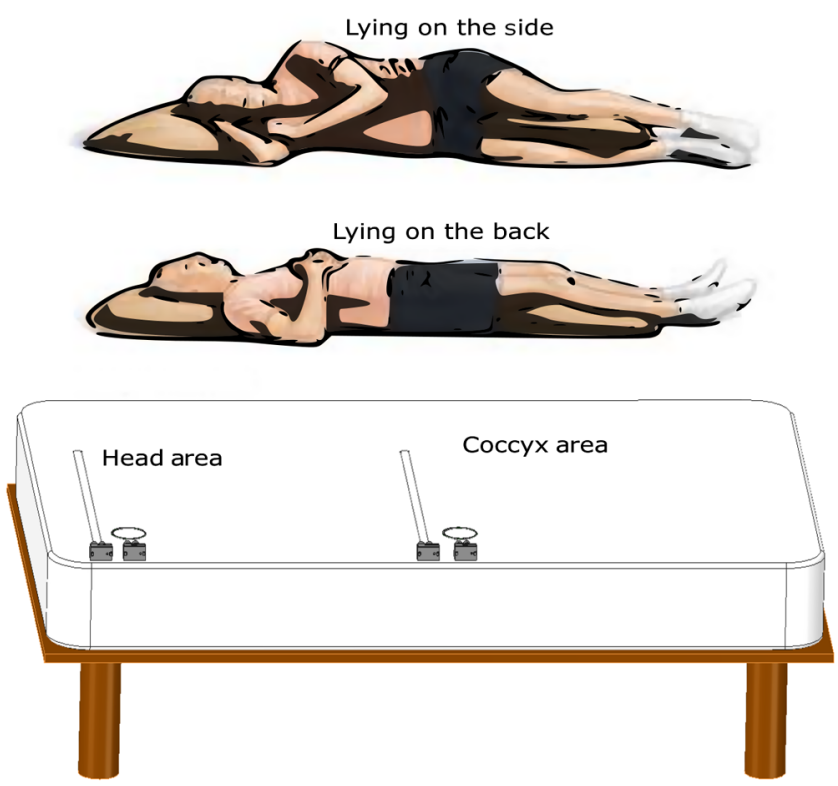

Fig. 3 Different patient's positions and sensor's locations during the different experiments. The pulse oximeter was placed in one of the fingers. under the coccyx area (one lying on their back and one lying on their side) and two measurements were taken under the head area (one lying on their back and one lying on their side) Fig. 3.

Both sensors were covered by a bedsheet and the distance between the closest point of the head and the closest point of the band sensor was $\sim 5 \mathrm{~cm}$ through the pillow (vertical direction), whereas the coccyx and the band sensor were only divided by the thickness of the sheet and the patient's clothes. The ring sensor was placed between 20 and $10 \mathrm{~cm}$ away from both coccyx and head areas (horizontal direction) depending on the size of the subjects. In order to simulate a deep sleep state before the beginning of the measurements, a little period of time was spent for the person to become relaxed and quiet. Moreover, the person in charge of the experiment was taking care of the correct development of the test and any movement was registered in a document. Then some additional measurements were taken under real sleeping conditions for longer periods of time, in order to verify the sensor and the processing method.

\subsection{Specklegram Processing}

The specklegram sensor produces an intensity change value for every frame, representing the perturbation produced between the two frames or time instants. The first step in every processing method is the differential processing summarized in Eq. (5) (Fig. 1). From the specklegram frames captured on the CCD, only the values of $\Delta I_{T}$ are needed. These sequential values are used to produce a one-dimensional and time-dependent intensity change signal. This signal summarizes all the speckle perturbation information needed for further processing.

\subsection{Inline Specklegram Processing}

The one-dimensional signal obtained has to be interpreted by transforming the intensity value into a time-dependent HR signal. In addition, this transformation has to take place in near real-time conditions (inline processing), transforming the perturbation value into HR value and motion detection with the least possible time delay and acquiring the best possible precision and accuracy.

The inline processing method uses a time lapse of some previous points of prestored intensity signal to estimate the HR of the patient for the current instant. These data are stored in a buffer, whose size must be defined according to the expected performance.

An inline measurement system should be capable of acquiring data fulfilling two requirements regarding time acquisition: duration of a single pulse, so as not to miss the pulses, and frequency of pulses, expressed in beats per minute (BPM).

- The duration of a single beat is around half a second. With a CCD camera producing 30 frames per second, this leaves around 15 points for sampling a single beat. 

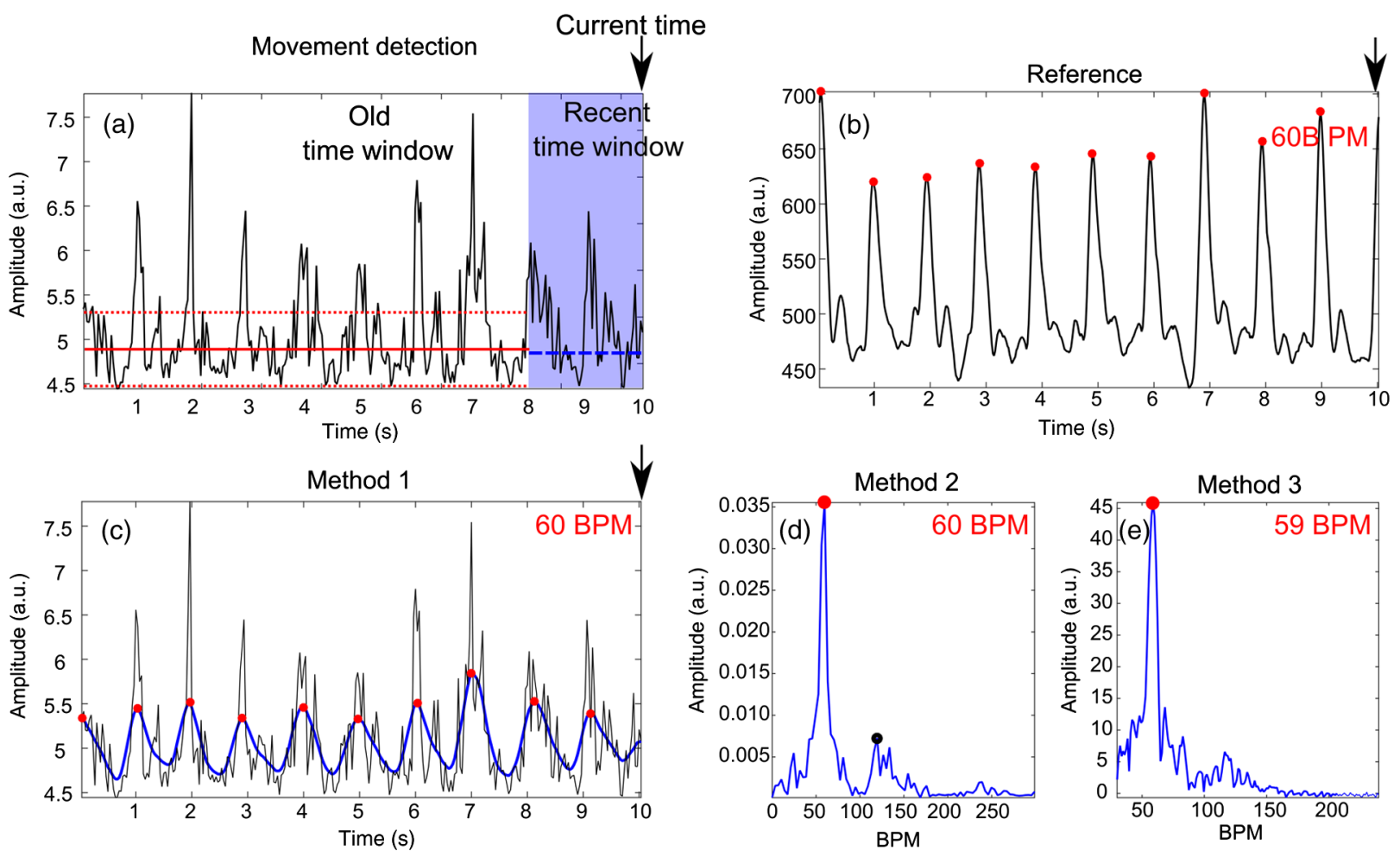

Fig. 4 Inline processing of current time (black arrow) applying a 10-s window buffer. (a) Original signal and movement detection mean values. Blue dashed line represents the recent time mean value. Red dotted line represents old time mean value. Dashed red is the $10 \%$ trigger threshold. (b) Pulse oximeter reference. (c) Method 1: original time signal (black thin) and smoothed (blue thick). (d) Method 2: Fourier transform of the first derivative. (e) Method 3: convolution by sinusoid. For every method (b to e), the obtained instantaneous heart rate is displayed in red numbers.

- Regarding the frequency of pulses, a standard heartbeat is around 55 to 75 BPM in healthy young people, but it can be lower than $40 \mathrm{BPM}$ in the case of athletes or patients with a heart disease. If we consider a worst case scenario of $20 \mathrm{BPM}$, this is one beat every $3 \mathrm{~s}$. If a minimum of two beats have to be stored to calculate the differential time interval, the buffer should be greater than $6 \mathrm{~s}$. When more points are stored, the system becomes more robust in case one or more pulses are not correctly detected. On the contrary, the longer the window, the more delayed the response; as for each instant, the information being displayed depends on a measurement not only from this very moment but also from events in the past. In this work, $10 \mathrm{~s}$ has been set as the buffer window providing $40 \%$ more data than needed in the worst case, but not increasing the output delay too much.

Once the buffer is full, the system can start processing the current instant with the information of the previous $10 \mathrm{~s}$ contained in this buffer.

\subsubsection{Motion detection}

This step is devoted to analyzing the amount of movement detected by the specklegram sensor, not only for the detection of motion but also to detect the time lapses when the HR will be wrongly measured due to motion. The buffer size is divided in two sections: the recent time window, comprehending the $20 \%$ most recent points, and the oldest time window, comprehending the previous $80 \%$ [Fig. 4(a)]. Both sections are averaged by the number of points in each section in order to obtain the mean intensity level of the recent and old status. The ratio among recent and old windows takes values near 1.0 when the signal is similar, but it varies strongly when there is an intense movement. If the ratio deviates more than the established threshold, movement detection is triggered. This threshold is to be set based on the required sensitivity.

\subsubsection{Heart rate measurement}

Different approaches have been considered for BPM estimation from the original one-dimensional data obtained from the speckle pattern, here noted as $X$. Processing time has to be considered, as the implementation has to provide the BPM measurement as close to real time as possible. As mentioned previously, a conventional pulse oximeter is used to obtain reference values [Fig. 4(b)].

Method 1: filtered signal and peak detection. This is the most straightforward approach [Fig. 4(c)]. Calculating the time lapse between two pulses, $T$, the frequency in Hertzs can be calculated as the inverse: $F=1 / T$, and expressed in BPM as $60 / T$. The signal obtained with the specklegram sensor is very sensitive and noisy, so a 10-point moving average filter is applied to this signal to reduce noise. After this step, peaks are detected and the median time between pulses $(i), T m$, is obtained.

$T m=\operatorname{median}\left(T_{i}\right)$ 

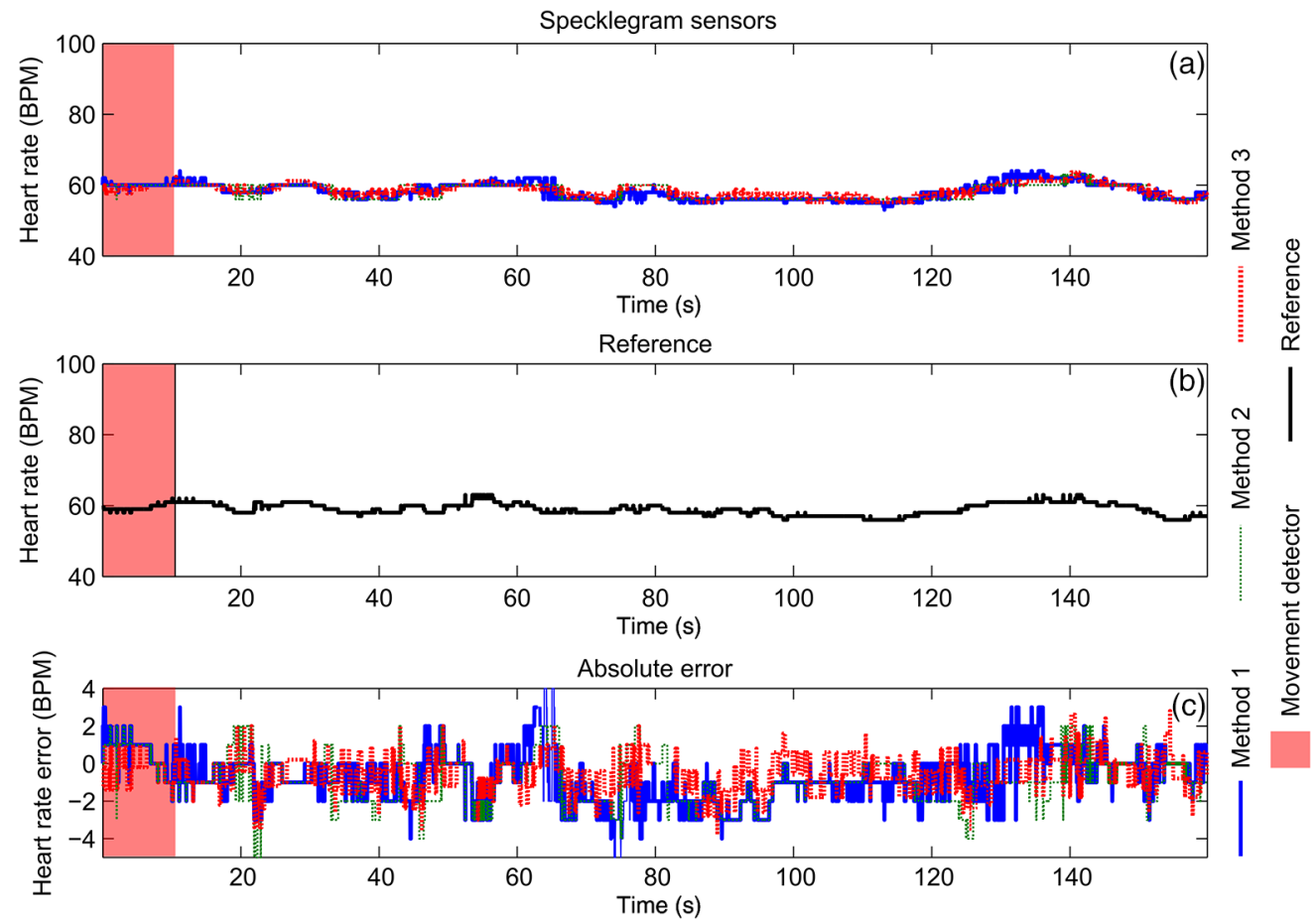

Fig. 5 Inline processing result for one of the patients for a measurement of $160 \mathrm{~s}$, lying on the back and band sensor placed under the head. (a) Heart rate estimated with the tree proposed methods. (b) Pulse oximeter reference. (c) Error for each of the three methods with respect to the reference. The reddish delimited region corresponds to movement detection active window, computed with the original signal. Note that it is active due to the signal variation at the start of the experiment.

Then the $H R$ in BPM is obtained as

$H R_{1}=60 * \frac{1}{T m}$.

Method 2: first derivative and Fourier transform. The first derivative is used to remove the offset of the original signal, not affecting the peaks present in the signal. Then a 10-point moving average filter is applied to avoid high-frequency noise, and the fast Fourier transform (FFT) is then computed. The FFT frequency presenting the maximum component corresponds to the heartbeat, expressed in BPM [Fig. 4(d)].

$H R_{2}=f_{\max (\operatorname{FFT}\{X\})}$.

Method 3: convolution by known frequency signal. First, a 10-point moving average filter is applied to this signal to reduce noise. Then, the signal is convoluted with sinusoids of different frequencies. The convolution result will be maximum when the specklegram sensor signal and the sinusoid present the same frequency [Fig. 4(e)]. The frequency sweep $\left(f_{c}\right)$ increases from $0.33 \mathrm{~Hz}$ (to detect $20 \mathrm{BPM}$ ) to $4 \mathrm{~Hz}$ (to detect 240 BPM). The different sinusoids are stored in advance to reduce inline computation time.

$S_{c}=\sin \left(2 \pi f_{c} t\right)$,

$H R_{3}=\max \left(X * S_{c}\right)$,

where $S_{c}$ represents the different computed sinusoids of different frequencies.

\subsubsection{Error estimator}

The heart rate obtained with the three proposed methods is compared with the reference. Two error metrics are used: mean absolute error and time percentage error.

- Mean absolute error is used to summarize the heart rate accuracy through the full-time measurement. It is computed by subtracting for each time instant the measured heart rate and the reference heart rate. The difference is obtained in absolute value and averaged for the duration of the measurement. The longer the measurement takes, the more the measurements there are to average, consequently, error is reduced.

- Time percentage error is computed to provide a durationinvariant error metric. The percentage error is computed to test instant accuracy. When the methods and reference differ $>10 \%$ of the reference BPM, this instantaneous measurement is considered erroneous. For the whole measurement, the number of frames producing an erroneous heart rate is computed as a time percentage of the total number of frames.

\section{Results and Discussion}

\subsection{Comparison of Methods}

The three processing methods are tested and compared with the reference for every combination of sensor, location, and patient's position (Fig. 5). From the specklegram sensor, the explained specklegram processing is applied to obtain the timedependent speckle variation signal. Then the inline processing 



Fig. 6 Inline mean heart rate summary for all the possible combinations of sensor, location, and processing methods. Ring sensor lying on the side (a), (e) and on the back (b), (f) is compared with band sensor lying on the side (c), (g) and lying on the back (d), (h).

methods make use of this preprocessed signal to obtain the heart rate in BPM.

The performance (in terms of error) is tested for each method, sensor, fiber optic positioning, and location. The recorded $160 \mathrm{~s}$ measurements are processed frame by frame, mimicking real-time acquisition. The mean heart rate obtained instantly can be compared in Fig. 6. The location under the head provides mean heart rate values nearer to those obtained with the pulse oximeter reference.

The absolute error is also computed for every instant, producing for every patient a mean absolute error value (Fig. 7).

The absolute error is affected by the duration of the measurement. However, the time error percentage metric in Table 1 shows the portion of frames presenting a measured heart rate that do not agree with the reference (as explained in Sec. 2.2.3). Measurements under the head location have better instant precision and even more in the case of lying on the side. Both ring sensor and band sensors get better results using processing method 1. Also, method 3 performs satisfactory with the band sensor. In those cases, error is committed less than $3 \%$ of the time (underlined values in Table 1).

Coccyx-located sensors receive intense heart bumps as this area is close to the heart; nonetheless, it is highly influenced by other body movements with higher displacements such as the ones from the abdominal region and breathing. This fact generates intense levels of noise and can produce periodic noise patterns on many occasions.

On the other hand, breathing and abdominal movements have lower intensity in the head area. Moreover, some authors have measured the intrinsic motion of the brain due to heart bumps, consisting of a rapid displacement in systole, with slow diastolic recovery. ${ }^{19,20}$ Those two conditions may explain why the head location produces better results than the coccyx location.

Under real scenarios, head location presents some advantages in comparison with the coccyx location or other locations. ${ }^{10}$ Placing this very soft sensor under the pillow means



Ring sensor


Band sensor
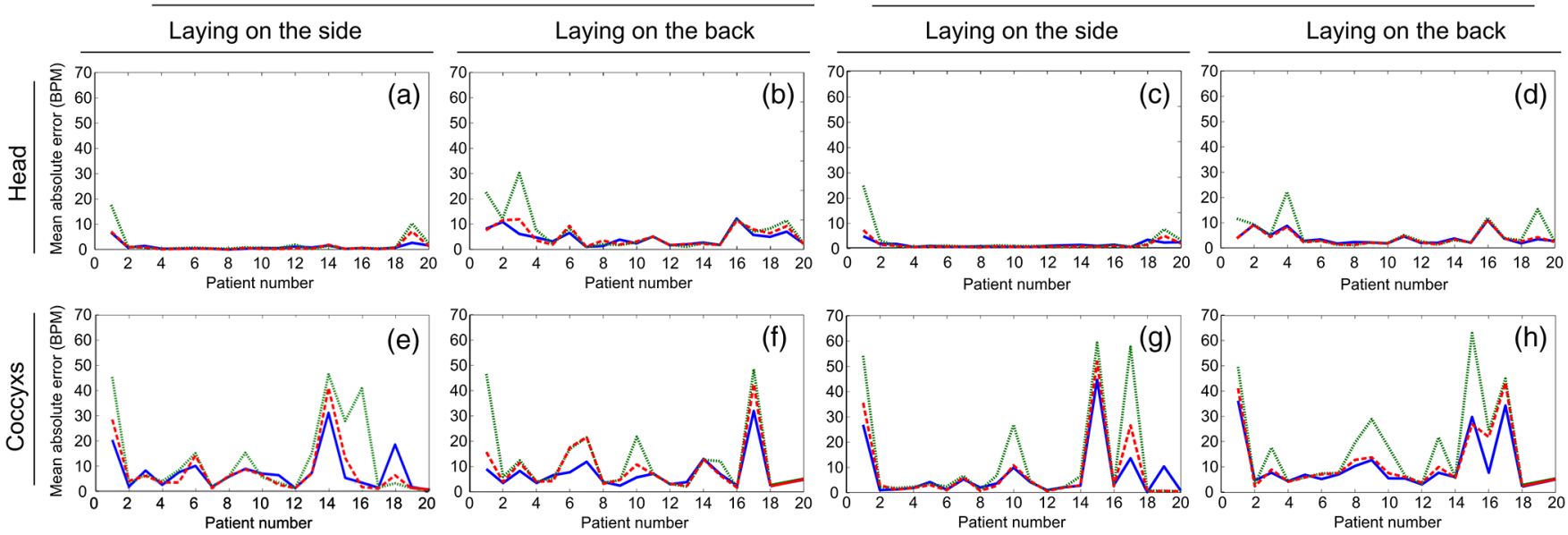

-Method $1 \quad$ m...'Method 2

$=-$ Method 3

Fig. 7 Inline mean absolute error summary for all the possible combinations of sensor, location, and processing methods. Ring sensor lying on the side (a), (e) and on the back (b), (f) is compared with band sensor lying on the side (c), (g) and lying on the back (d), (h). 
Table 1 Heart rate time percentage error for all the combinations.

\begin{tabular}{|c|c|c|c|c|c|c|c|c|}
\hline \multirow[b]{3}{*}{ Processing method } & \multicolumn{4}{|c|}{ Head } & \multicolumn{4}{|c|}{ Coccyx } \\
\hline & \multicolumn{2}{|c|}{ Side } & \multicolumn{2}{|c|}{ Back } & \multicolumn{2}{|c|}{ Side } & \multicolumn{2}{|c|}{ Back } \\
\hline & Ring & Band & Ring & Band & Ring & Band & Ring & Band \\
\hline 1 & $2.48 \%$ & $2.09 \%$ & $27.78 \%$ & $20.21 \%$ & $30.89 \%$ & $24.60 \%$ & $38.19 \%$ & $49.67 \%$ \\
\hline 2 & $7.41 \%$ & $6.45 \%$ & $31.02 \%$ & $22.66 \%$ & $33.95 \%$ & $26.00 \%$ & $45.53 \%$ & $59.29 \%$ \\
\hline 3 & $4.15 \%$ & $2.91 \%$ & $27.14 \%$ & $18.58 \%$ & $25.69 \%$ & $20.64 \%$ & $40.68 \%$ & $52.90 \%$ \\
\hline
\end{tabular}

Best performances are bold faced.

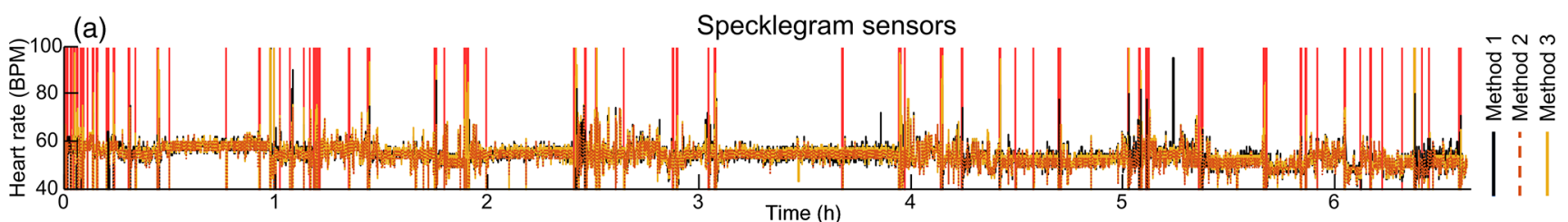

(b)

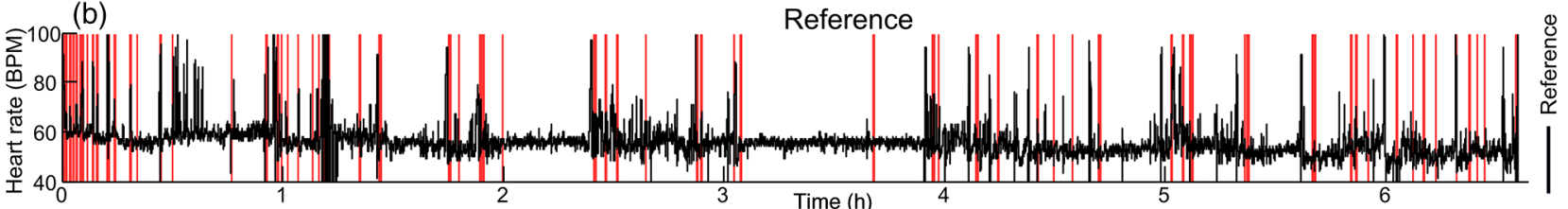

(c)



Fig. 8 Inline processing result for a long-term measurement under the head of the patient. The motion triggered in (c) is overlaid in (a) and (b) for comparison. This trigger implies that there is movement in the following frames, and hence, heart rate will not be accurate in the affected frames.

that the patient will not notice its presence or interfere with it when accommodating (sitting, rolling, or reclining), therefore, it will not produce any inconvenience of any kind. Third, it is this configuration that allows the sensor to be less exposed to damage caused by scratches, hits, strains, or other mechanical movements, as well as damage caused by body fluids. All these advantages highlight this technology to be suitable for an implementation not only in the hospital but also in scenarios such as geriatrics or patient's homes.

Computation time for the three methods has been measured in a MATLAB implementation (MATLAB R2013b 64 bits, processor Intel core $15-3570,8 \mathrm{~Gb}$ RAM), resulting in a mean computation time for each frame in the three proposed methods of $0.25,0.35$, and $38.0 \mathrm{~ms}$, respectively.

\subsection{Best Method for Long-Term Test}

After the evaluation of all the combinations, the option of band sensor located under the head and lying both on the back and in a lateral position is tested with a long-term measurement during sleep (Fig. 8). This measurement shows more active triggers of the movement detector.

When the movement detector is triggered, it can be seen how the reference and the processed heart rate measurements vary strongly. The three processing methods correlate well with the reference, as shown in Table 2.

According to Table 2, the mean absolute error when comparing the proposed methods with the reference is below $6 \mathrm{BPM}$ (which represents around 10\% error), and at most, $22.20 \%$ of the measurement is incorrect. It has to be taken into account that this does not mean failure, but that there is motion affecting the measurement. The accuracy is reduced, due mainly to the fact that this was a real condition, without any control or special

Table 2 Mean heart rate and errors for the long-term sleep measurement under the head.

\begin{tabular}{lccc}
$\begin{array}{l}\text { Processing } \\
\text { method }\end{array}$ & Heart rate (BPM) & $\begin{array}{c}\text { Mean absolute } \\
\text { error (BPM) }\end{array}$ & $\begin{array}{c}\text { Time percentage } \\
\text { error }\end{array}$ \\
\hline 1 & 54.04 & 4.89 & $20.65 \%$ \\
2 & 52.98 & 5.35 & $22.20 \%$ \\
3 & 53.61 & 4.94 & $21.06 \%$ \\
Reference & 56.07 & & \\
\hline
\end{tabular}




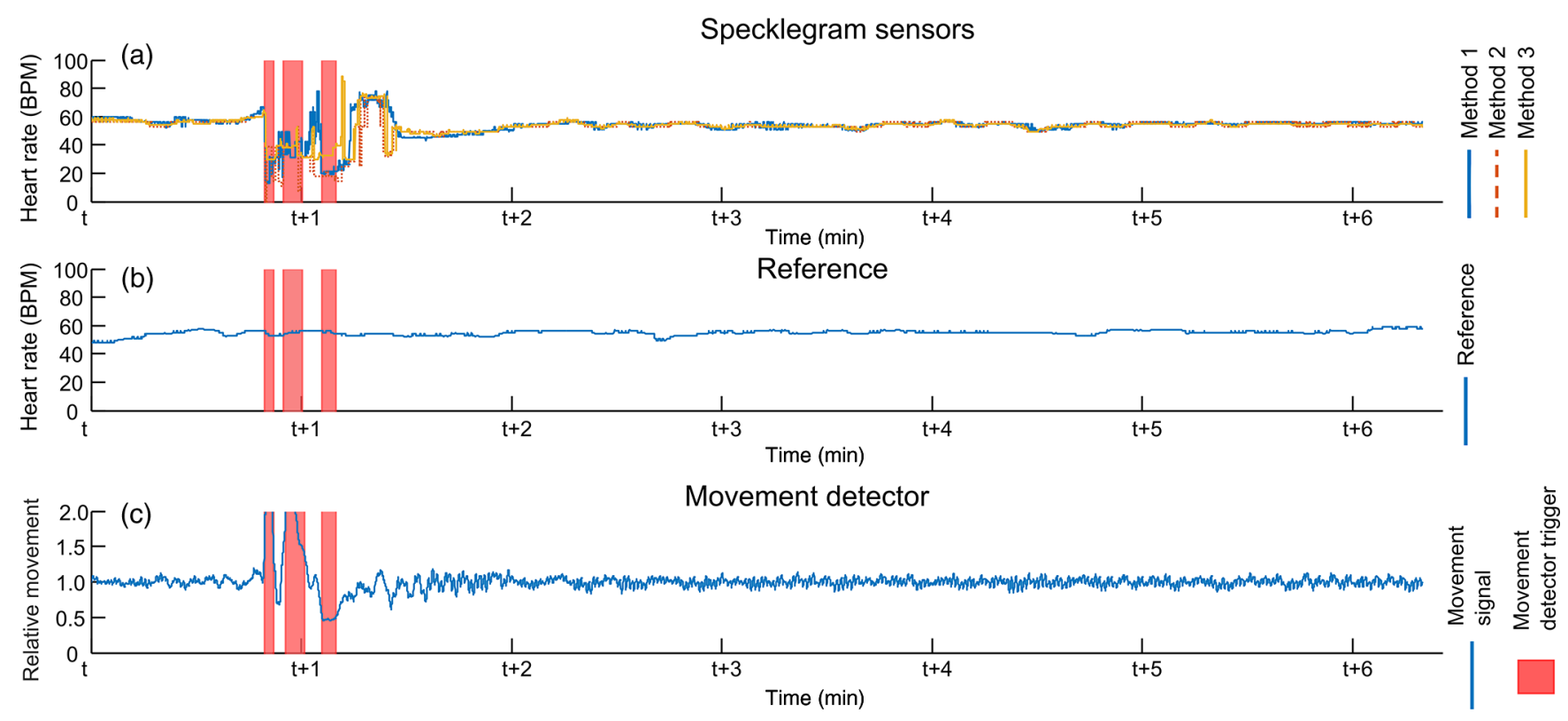

Fig. 9 Zoom in of Fig. 8 when time $t=3 \mathrm{~h}, 3 \mathrm{~min}$, and $39 \mathrm{~s}$, presenting a calm interval after a movement.

care during the measurement in order to simulate real operating conditions. Also, the variation of the patient's position (lying on the back or on the side) affects the result, detecting the position changes or any other movement during sleep.

In Fig. 9, a short portion of the same measurement in Fig. 8 is displayed for visual comparison. When the person under analysis is in deep sleep and does not present movements, the performance is similar to that observed during the method's comparison experiments.

\section{Conclusion}

A noncontact monitoring system has been implemented and evaluated in this work. Based on the obtained results, it is possible to conclude that a fiber optic specklegram sensor exhibits the potential to be employed as a continuous activity monitoring device, in this case for heart rate measurement and motion detection tool, achieving high accuracy.

Among the multiple options that have been studied, the results suggest that a stretched fiber optic configuration under the person's head is the optimal sensor design and location. When it comes to the processing method, the three tested methods exhibited satisfactory results, being the smoothed signal, the fastest method, and the one that offers the best results. Eventually, the combination of the optimal spatial distribution and the optimal processing method shows accuracies below $3 \%$ instantaneous error and heart rate mean difference with the reference below $10 \%$ BPM.

As opposed to most of the current monitoring methods, this specklegram system is a low-cost and widely applicable tool. This adds to the simplicity and robustness of measurements, and supports the potential for being used as a basic monitoring system in all the hospital rooms and local environment.

\section{Disclosures}

No conflicts of interest, financial or otherwise, are declared by the authors.

\section{Acknowledgments}

This work has been supported by the Spanish Government through the Ministry of Economy and Competitiveness projects TEC2013-47264-C2-1-R and TEC2016-76021-C2-2-R.

\section{References}

1. P. C. Richardson, "The insulated electrode," in Proc. of the 20th Annual Conf. on Engineering in Medicine and Biology, Boston, Massachusetts, p. 157 (1967).

2. W. Chaboyer, "Predictors of adverse events in patients after discharge from the intensive care unit," Am. J. Crit. Care 17(3), 255-263 (2008).

3. M. P. Young et al., "Inpatient transfers to the intensive care unit: delays are associated with increased mortality and morbidit," J. Gen. Intern. Med. 18(2), 77-83 (2003).

4. D. Teichmann et al., "Non-contact monitoring techniques-principles and applications," in 2012 Annual Int. Conf. of the IEEE Engineering in Medicine and Biology Society (EMBC), Annual Int. Conf. of the IEEE (2012).

5. L. A. Aarts et al., "Non-contact heart rate monitoring utilizing camera photoplethysmography in the neonatal intensive care unit-a pilot study," Early Hum. Dev. 89(12), 943-948 (2013).

6. S. Kwon, H. Kim, and K. S. Park, "Validation of heart rate extraction using video imaging on a built-in camera system of a smartphone," in 2012 Annual Int. Conf. of the IEEE Engineering in Medicine and Biology Society, IEEE (2012).

7. L. Tarassenko, "Non-contact video-based vital sign monitoring using ambient light and auto-regressive models," Physiol. Meas. 35(5), 807-831 (2014).

8. L. Dziuda, "Fiber-optic sensors for monitoring patient physiological parameters: a review of applicable technologies and relevance to use during magnetic resonance imaging procedures," J. Biomed Opt. 20(1), 010901 (2015).

9. W. B. Spillman, Jr. et al., "A 'smart' bed for non-intrusive monitoring of patient physiological factors," Meas. Sci. Technol. 15(8), 1614-1620 (2004).

10. P. Podbreznik et al., "Cost-efficient speckle interferometry with plastic optical fiber for unobtrusive monitoring of human vital signs," J. Biomed. Opt. 18(10), 107001 (2013).

11. M. Lomer et al., "Speckle POF sensor for detecting vital signs of patients," Proc. SPIE 9157, 91572I (2014).

12. L. Rodriguez-Cobo, "Speckle characterization in multimode fibers for sensing applications," Proc. SPIE 8413, 84131R (2012). 
13. J. Škrabec et al., "Preliminary detection of periodic perturbations using speckle imaging and interframe gradient," in Recent Advances in Applied and Biomedical Informatics and Computational Engineering in Systems Applications (AIC 2011) (2011).

14. W. Spillman et al., "Statistical-mode sensor for fiber optic vibration sensing uses," Appl. Opt. 28(15), 3166-3176 (1989).

15. T. Francis et al., "Submicrometer displacement sensing using innerproduct multimode fiber speckle fields," Appl. Opt. 32(25), 4685-4689 (1993).

16. S. Yin, P. Purwosumarto, and T. Francis, "Application of fiber specklegram sensor to fine angular alignment," Opt. Commun. 170(1), 15-21 (1999).

17. L. Rodriguez-Cobo et al., "Optical fiber strain sensor with extended dynamic range based on specklegrams," Sensors Actuators A 203, 341-345 (2013).

18. Z. Zhang and F. Ansari, "Fiber-optic laser speckle-intensity crack sensor for embedment in concrete, Sensors Actuators A 126(1), 107-111 (2006).

19. D. Greitz et al., "Pulsatile brain movement and associated hydrodynamics studied by magnetic resonance phase imaging," Neuroradiology 34(5), 370-380 (1992).

20. B. P. Poncelet et al., "Brain parenchyma motion: measurement with cine echo-planar MR imaging," Radiology 185(3), 645-651 (1992).

Alberto Rodríguez-Cuevas graduated in 2015 as a chemical engineer. He received his master's degree in chemical engineering from the Universidad de Cantabria. He is currently working as a researcher at the Photonics Engineering Group. His research interests are mainly focused on optical fiber sensors for different scenarios.
Eusebio Real Peña graduated in 2012 as a telecommunications engineer. He received his master degree in Information and Communication Technologies both from the University of Cantabria. $\mathrm{He}$ is currently working toward his $\mathrm{PhD}$ in telecommunications, focused on biomedical imaging and biosignals processing.

Luis Rodríguez-Cobo received his BSc and MSc degrees in telecommunication engineering from the University of Cantabria, Santander, Spain, in 2009 and 2010, respectively. He obtained his $\mathrm{PhD}$ in telecommunication engineering at Photonics Engineering Group of the University of Cantabria in 2013. His research interests are mainly focused on optical fiber sensors for different scenarios and he has coauthored more than 25 ISI journal papers and collaborated in different $R \& D$ project.

Mauro Lomer received his $\mathrm{PhD}$ in electronics from the Université de Limoges, France, in July 1992. His doctoral work is based on the study of whispering gallery modes and fabrication nonlinear optical waveguide. Since October 1992, he works as associate professor in the University of Cantabria in the Photonic Engineering Group. His current research interests include polymer optical fibers and fiber optic speckle phenomenon.

José Miguel López Higuera received his telecommunication technical engineering degree from the Universidad Laboral de Alcalá de Henares, Madrid, Spain and his $\mathrm{PhD}$ in telecommunication engineering, from Universidad Politécnica de Madrid (UPM), Madrid. He is the founder and the head of the Photonics Engineering Group, TEISA Department, University of Cantabria, Spain. He has directed more than 50 R\&D projects and has written or cowritten more than 400 publications. 